\title{
Charging and Discharging a Coaxial Borehole Heat Exchanger as a battery
}

\author{
Hongshan Guo ${ }^{1,2}$, Forrest Meggers ${ }^{1,2}$ \\ ${ }^{1}$ Princeton University, Princeton, NJ, United States \\ ${ }^{2}$ Andlinger Center for Energy and the Environment, Princeton, NJ, United States
}

\begin{abstract}
We investigated the possibility of using deep coaxial borehole heat exchanger as a potential storage device of not only heat or coolness, but also an invaluable device that shifts the peak load of both heating and cooling for an electric system. We want to, therefore, investigate the potential of using CBHE as a battery. Using a bottom-up approach, we analyzed the existing literature and adapted the existing model with the sole emphasis on the thermal improvements of deeper borehole when considering the impact of geothermal gradient. This paper presents some preliminary analysis of how CBHE configuration can be used to maximize its performance as an energy storage option. Future work on improving the accuracy of the model, validate with data from an actual borehole as well as conducting multi-variate rigorous optimization could further improve the current study.
\end{abstract}

\section{Introduction}

The geothermal gradient is usually a component that is overlooked for shallower geothermal applications when it comes to building system designs as per DiPippo (2004) - wells are often drilled shallower since the amount of heat extraction is limited by the depth that the well can reach, or in the case of using a borehole heat exchanger (BHE): the maximum of the temperature of soil it can extract fromLadislaus Rybach and Robert J. Hopkirk (1995).

Some recent studies have pointed out that increasing the depths of the wells may potentially lead to a higher temperature at the bottom of the borehole and hence making the boreholes more suitable for charging and discharging under different operating scenarios, for example, both heating and cooling at the same day during a swing season GUILLAUME (2011). The resulting temperature fluctuation at the borehole wall needs to be considered as a part of objective function when trying to optimize for a hypothetical BHE. The coaxial borehole heat exchanger (CBHE) configuration is known to be able to harvest the maximum amount of heat - particularly when the inner pipe is insulated. We present a novel approach to analytically simulate the vertical temperature distribution in such a CBHE concerning different heat injection/extraction under transient conditions as shown by Palm et al. (2014).
Conventionally, the method to evaluate the temperature distribution inside a CBHE is very limited to either doing the simulation in computational fluid dynamics simulation by segmenting the entire fluid regime into different finite volumes, or finite element modeling of flow using algorithms such as FEFLOW. Very few researchers have worked on providing an analytical solution for the heat transfer from the fluid inside coupled with the geothermal gradient in the meantime.

We have adapted, for this paper, a novel analytical approach that was first developed by Beier et al. (2013), which solves for the vertical temperature profile at different depths inside the borehole for any given combination of CBHE configurations and flow rates. We were, therefore, able to calculate the added thermal benefit from having a borehole that has larger depth and a lower flow rate. More importantly, this means added storage capacity for heat/coolness, as the transient increase/decrease of temperatures' disruption towards the borehole wall temperature at every single time step of the simulation without sophisticated segmentation of the overall CBHE geometry Palm et al. (2014).

We have completed the analytical model and were able to simulate different borehole wall temperature during stages of constant heat injections (model from Acuña and Palm (2013) as well as variable temperature injections Palm et al. (2014). The fluctuation from added heat extraction appears to be providing a less pronounced curve of vertical temperature distribution, which appears to be supporting our hypothesis of CBHEs being fit for rapid charge/discharge during energy storage. We are in the process of expanding our analysis towards more configurations and geothermal gradients in the borehole to provide a more comprehensive set of data for further analysis and are expecting to provide those discussions in the near future.

\section{Methods}

\section{Analytical model}

Adopting the analytical model first developed by Beier et al. Beier et al. (2013) and incorporating the methods used in a transient heat exchange analysis Palm et al. (2014), we were able to establish a bottom-up model that describes the heat exchange in- 
side a coaxial borehole heat exchanger within its inner pipe, annulus, and the surrounding soil. The nondimensionalization of the temperature Equation 1 from central tube $\left(T_{D 1}\right)$, annular pipe $\left(T_{D 2}\right)$ and soil $\left(T_{D S}\right)$, while the radial distance from the centre, distance from ground surface and time can all be nondimensionalized following Equation 2,3 and 4 to have the heat transfer solved first in the Laplace Domain before being converted back to the real-time scale through Inverse Laplace Transformation. The thermal conductivity and heat capacity of soil $k_{S}, c_{S}$ as well as the well configuration follows Beier's studies such that the experimental data in the original study can be used to validate the new model.

$$
\begin{gathered}
T_{D}=\frac{T-T_{r s}}{T_{i n}-T_{r s}} \\
r_{D}=\frac{r}{r_{e o}} \\
z_{D}=\frac{z}{L} \\
t_{D}=\frac{k_{S} t}{c_{S} r_{e o}^{2}}
\end{gathered}
$$

Following the non-dimensionalization, the energy balance between the flow channels and the surrounding soil takes the form of Equation 5 and 6 .

$$
\begin{array}{r}
\frac{A_{D 1}}{2} \frac{\partial T_{D 1}}{\partial t_{D}}+\frac{\partial T_{D 1}}{\partial z_{D}}+N_{12}\left(T_{D 1}-T_{D 2}\right)=0 \\
N_{s} A_{D 2} \frac{\partial T_{D 2}}{\partial t_{D}}-\frac{\partial T_{D 2}}{\partial z_{D}}+N_{12}\left(T_{D 2}-T_{D 1}\right)+ \\
N_{s}\left(T_{D 2}-T_{S D}\right)=0
\end{array}
$$

Using boundary conditions at the top and bottom of the storage on top of the continuity equation of the flow, we obtained solutions for two flow channels. These boundary conditions may therefore be written as Equation 7, 8 and the far-field boundary condition in the radial direction that is the same as geothermal gradient in Equation 9.

$$
\begin{gathered}
T_{D 1}\left(0, t_{D}\right)=1 \quad t_{D}>0 \\
T_{D 1}(1, t D)=T_{D 2}(1, t D) \quad t_{D}>0 \\
\frac{\partial T_{D S}}{\partial z_{D}}\left(r_{D} \rightarrow \infty, t_{D}, z_{D}\right)=g_{D}\left(z_{D}\right) \quad t_{D}>0
\end{gathered}
$$

Solving the heat transfer inside the CBHE first under its Laplace Transformation as was the work from Palm et al. (2014), its solution is then brought back to the real-time domain by doing the inverse Laplace Transform with Stehfast Algorithm as suggested by Beier et al. (2013). Assuming a constant flow rate of $0.58 \mathrm{~L} / \mathrm{s}$ (also as used by Beier et al. (2013)), the resulting flow across the inner pipe and the annulus can be solved by introducing the resulting linear velocities and hydraulic diameters to obtain Reynolds number, Nusselt number as did Palm et al. (2014) to estimate the convective heat transfer coefficient at various flow/pipe interfaces as proposed by Gnielinski (1976). Using the boundary conditions from Equation 9,8 and 7 , it is, therefore, possible to solve for the individual temperature expressions for both the inlet and outlet flow paths, which when solved in the real-time domain needs to go through also the inverse Laplace Transformation.

The adapted model has clear limitations despite being verified against the original model and data with reasonable accuracy, that it lacks the second set of data to be validated against: although Palm et al. (2014) was able to achieve transient temperature modeling, we adapted this non-dimensionalize method for our study where the temperature injected is also assumed a known variable of the working fluid which interacts with the CBHEs. One additional difference is the selection of the reference temperature of the ground $T_{r s}$ was assumed to be 8.4 degree Celsius, as was defined by Beier et al. (2013). As we're considering a temperature distribution along the borehole depth, the ground temperature is no longer a constant one as assumed by the existing models, but should have minimal effect on the results that we can obtain from the model, as the reference temperature $T_{r s}$ is only used to reduce the computational costs for the temperatures that we can get from the model. Acknowledging the potential of increased computational time because of incorporating intense iterations, we will remain using the same temperature non-dimensionalize method shown in Equation 4 within the scope of this study.

\section{CBHE as a battery}

The concept of a (or several) deeper CBHE acting as a battery is both easy and difficult to understand: Easy being the clear thermal benefit of harvesting the warmer temperature closer to the bottom of the borehole, and dumping heat closer to the top. This same concept formed the basis of creating Figure 1. Essentially, it stores heat in summer by dumping heat to the bottom of the borehole and discharge the stored heat in winter by extracting it from the bottom through the inner pipe. More complex operations during the swing season can also be achieved through optimized dumping and extraction of heat to the borehole but is beyond the scope of this paper. To better reflect how the CBHE could have varying performance when the geothermal gradient varies, we also investigated how the geothermal gradient affects the resulting temperature distribution inside a CBHE. Since the most common geothermal gradient ranges from 1 Kelvin per 100 meters to 5 Kelvin per 100 metersRybach et al. (1992), we selected three different values to estimate the influence 


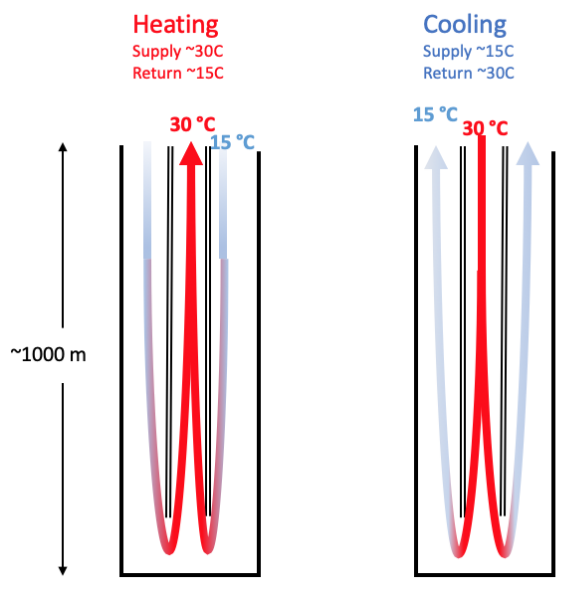

Figure 1: Conceptual drawing of CBHE and its functionality when used as a thermal battery for energy delivery systems.

of geothermal gradient on the resulting thermal performance of a CBHE. Unlike varying the inner pipe radius, pipe wall thickness as well as the piping materials, which change the shunt resistance (as shown in Figure 2) of the borehole, the geothermal gradient is a variable that is not subject to any changes in design when a project location is determined.

\section{Results}

We were able to produce a series of temperature distribution inside CBHEs with different geothermal gradients as well as different CBHE configurations. To better reflect the assumptions used in each case, we are plotting all the underlying assumptions in the corresponding legend used when generating the following figures. It is important to stress that the temperature profile of the borehole wall is updated at every single step such that the transient heat transfer between the borehole and the working fluid can be carried over to the next time step.

Changing the inner pipe thickness and its thermal conductivity resulted in the most obvious change of temperature distribution inside the CBHE, as was shown in Figure 3. When the thickness of the inner pipe decreases, the shunt resistance increases while the cross-sectional area of the inner pipe also decreases, which pushes the temperature drop in the inner pipe down to below 1.5 degree Celsius.

Assuming a more insulated inner pipe with a smaller cross-sectional area, i.e., lower temperature drop in the inner tube, Figure 4 shows the temperature distribution inside the CBHEs when depths vary assuming various geothermal gradients. It is essential to point out much of the heat extraction rate normalized by depths are extraordinarily high and unlikely to be designed since they not only require a significant amount of pumping power, but can also cause geothermal depletion rather quickly since the heat extraction normalized by depths are far much beyond the $50 \mathrm{~W} / \mathrm{m}$ that is commonly used to estimate the thermal potential of CBHEs during operation as pointed out by Acuña (2013). During a shoulder season where the demands of heating and cooling may be balanced within either a diurnal cycle or over an entire week, operating a CBHE-based system rapidly with different injection temperatures towards different depths - and could become an exciting future direction of research.

Varying the flow rate could also lead to significant variation of heat extraction rates, or as shown in Figure 5. Straightforwardly, the larger the flow rate, the larger the heat extraction rate, and the lower the temperature available at the outlet/bottom of the borehole. The heat exchange between the working fluid and the surrounding soil can be either enhanced by changing the flow regime through increasing the velocity or decreasing the cross-sectional area for the flow to move through, which coincide with the observations we made when changing the thickness of the inner pipe and holding the exterior diameter of the inner pipe and the outer tube the same. Also, we showed the temporal variation of the total extraction rate from a $\mathrm{CBHE}$ when keeping the injected water at 5 degree Celsius. The heat extraction rate fluctuation at the first two hours is due to the instability of the soil thermal resistance estimation for early-state operations of CBHE.

The investigation of the suitability of the CBHEs as batteries, on the other hand, also requires more detailed examination, specifically relating to the resulting temperature profile as assumed in Figure 1. The temperature at the top and bottom of the CBHEs will vary on a case-by-case basis, and requires more detailed investigation to satisfy project-specific needs, and could be investigated more thoroughly with a specific site and a set of geological conditions. Although we can show the resulting temperature profiles inside the CBHE to potentially illustrate the thermal potentials harvested from deeper wells, the resulting temperature distribution will remain placeholders before a more practical set of parameters backed with experimental data. The Beier dataset that we're currently using to verify our model is from a borehole that is 178 meters deep, including an 18meter deep top section. As we're attempting to verify the geothermal performance of deeper boreholes that are 1000 meters or even 2000 meters deep, this dataset alone is most likely not going to be enough to validate the applicability of the proposed model on deeper CBHEs. It is therefore highly desirable to obtain datasets that provide information of temperature distribution inside deep CBHEs with more considerable depths - preferably after more extended periods of operation, as the current Beier data only shows the results after 67 hours of operation. 


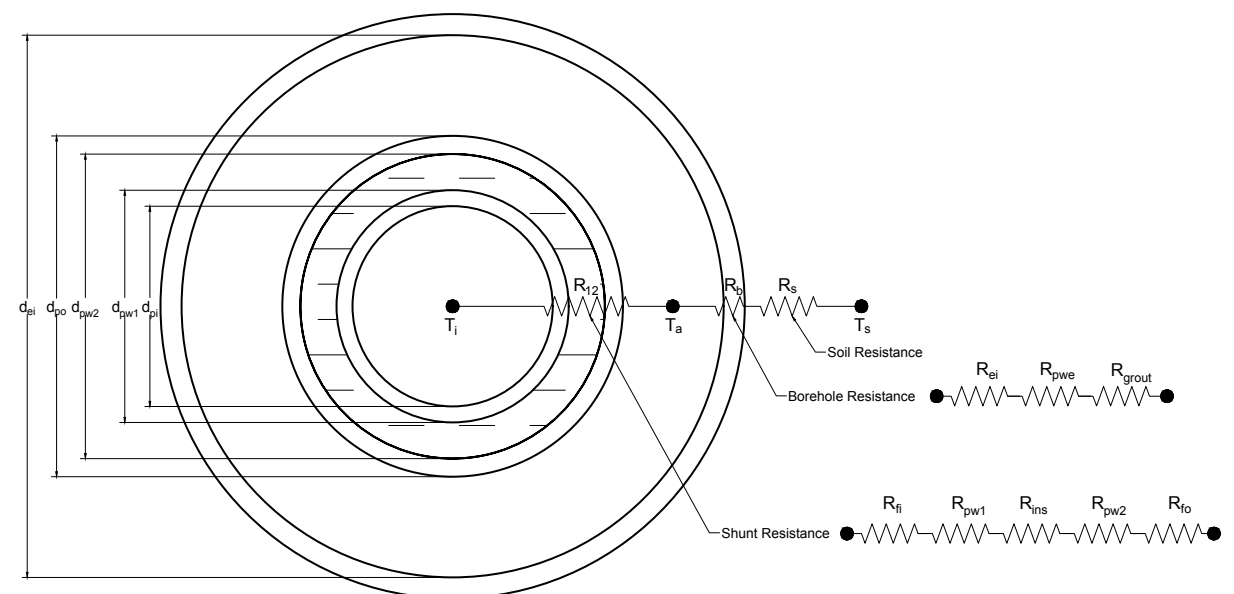

Figure 2: Cross-sectional diagram of how the thermal resistance of a CBHE can be calculated.
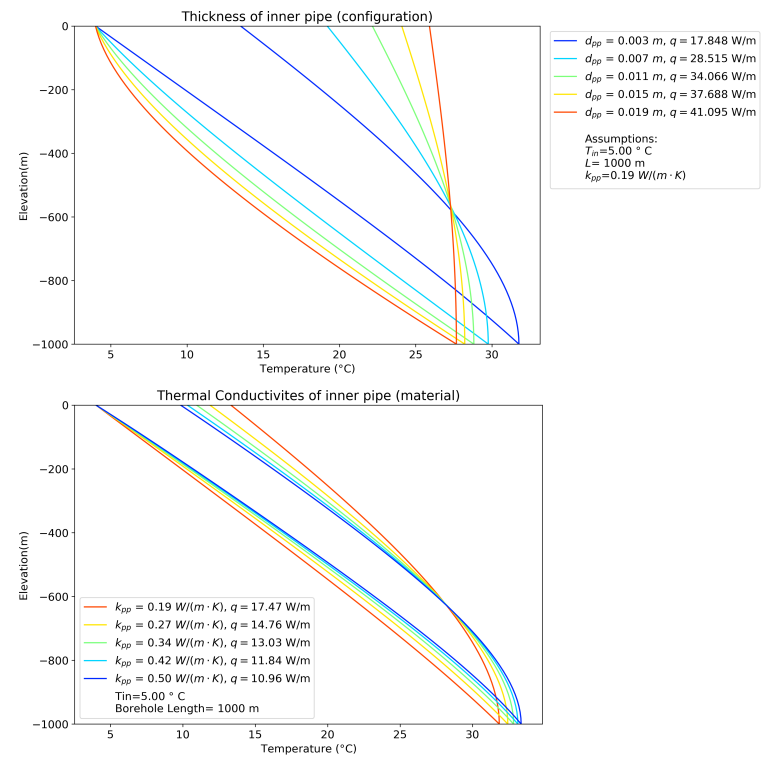

Figure 3: Temperature distribution along flow path variation with different levels of insulation for the inner pipe

\section{Discussion}

Starting from the analysis of the results that we have shown in this paper, it would be fascinating to question whether any of them may link to the capacity of CBHEs as batteries that can be used to optimize the grid performance. Using heating degree days and cooling degree days, for example, could help to generate a baseload profile as the load for the CBHEs. Using these load profiles, it is possible to estimate the resulting demand peak of both the heating in the winter and cooling in the summer, which can be mitigated by electrification of the CBHEs, as the boreholes can be charged with heat during the summer and strategically export these heat during cold winters. We may achieve this either with conventional electricitydriven heat pumps or even more drastically, through direct heat exchange between the borehole and the
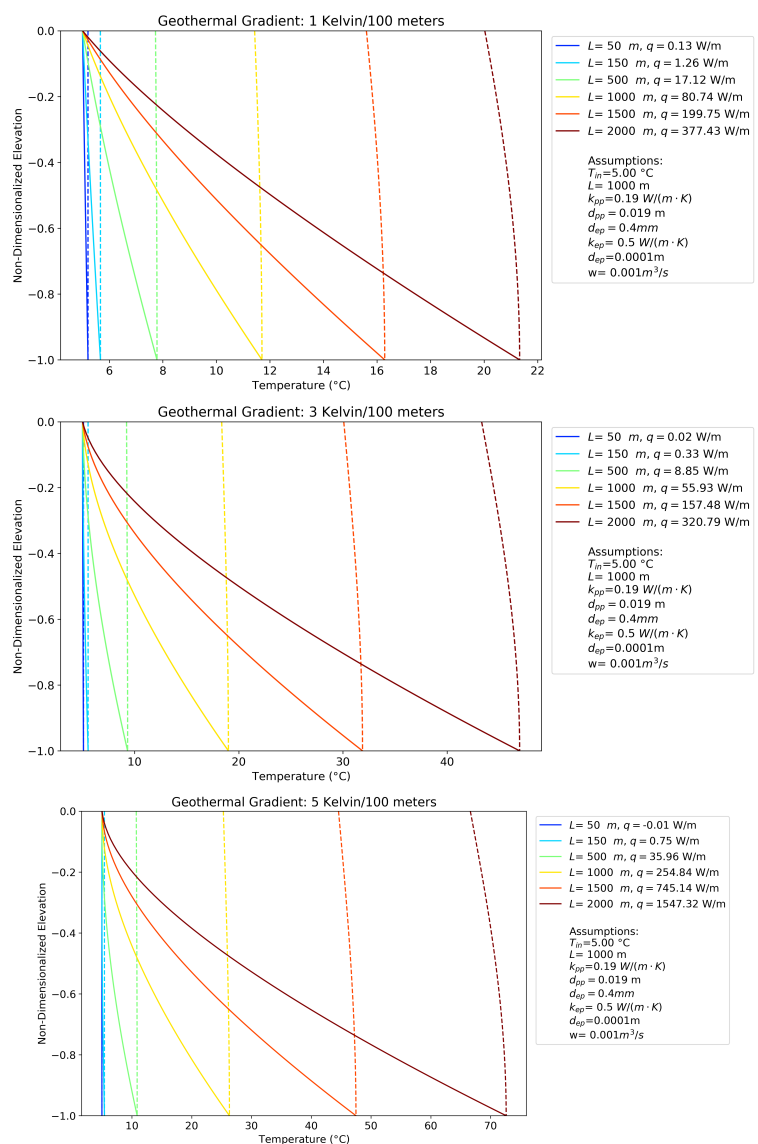

Figure 4: Temperature distribution variation with respect to different geothermal gradients and ample insulation on the inner pipe assumed

buildings via the working fluid.

The impact of using thermal energy storage, i.e., CBHEs that are deeper than conventional ones in this paper could apply to other use cases: the direct usage of heat from the bottom and the direct cooling from the top of CBHEs might be possible by arranging the flow rate or CBHE design accordingly. The electricity demand profile could, therefore, have their peaks 

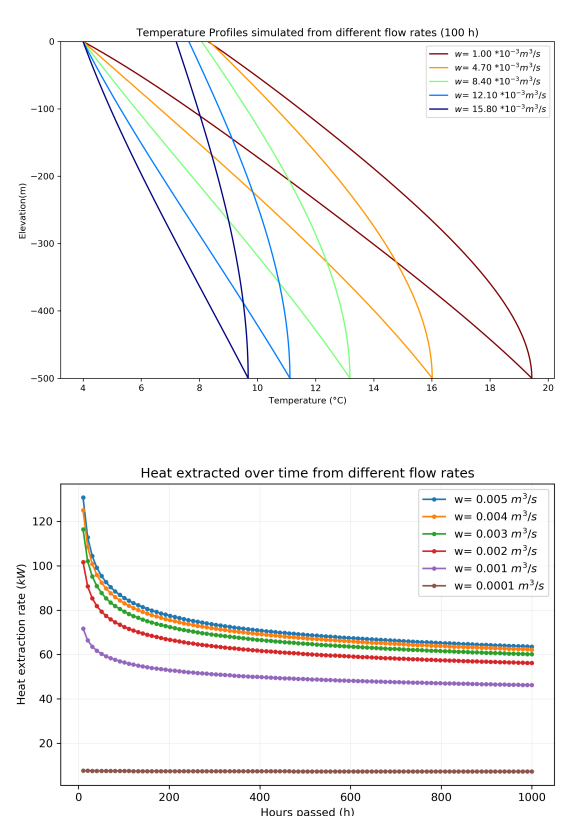

Figure 5: Temperature distribution along flow path variation with different flow rates and their resulting heat extraction rates simulated at the 100th hour (top) and total heat extraction change over time.

reduced and shifted, thus providing valuable reliefs for the grid during hours of high energy demands.

Future discussions on this topic could also focus on the simulation of the demand profile of both the heat pump and the direct use scenarios under different usage/occupancy profiles, i.e., residential, commercial or mixed-uses. The resulting temperature fluctuations, as well as the energy demand, may provide valuable insights to future system designs as well as guidance towards additional optimization efforts. Redistributing the heat or coolness through optimized thermal storage and thus reducing the electricity demand is only one possible usage of the proposed technology.

Modeling on how the temperature evolves, more precisely speaking on the feasibility of the usage of direct heating could also be investigated in future studies. According to a preliminary analysis that we have conducted on the potential COP of the resulting CBHE system, a small temperature lift on the supply side could suggest a resulting COP of up to 40 but needs more detailed modeling in future studies. The present study provides only theoretical and preliminary investigations towards possible operation scenarios for a CBHE when varying its configuration and overall design parameters, and maybe further improved with more realistic flow rates to reach better simulation results with more realistic load profiles.

The pumping power and drilling costs could potentially also be an exciting future research topic. An analysis on estimating the return on investment
(ROI) or life cycle assessment (LCA), for example, could significantly increase the likelihood of this research to be applied in real funded projects. Such economic or enviro-economic analysis should help not only evaluating deeper CBHEs among renewable technologies, but also elicit interests among other researchers to build similar CBHEs, thus provide more datasets from different locations and geological makeups around the world.

This analytical model may not be as precise as a numerical model that solves the heat transfer at every node/finite volume but requires significantly reduced computational time. As we set up the Python-ready class and have it initiated for each configuration, the simulation of the resulting temperature distribution profile (including and not limited to the temperature at the inlet, outlet, and the immediate surrounding soil) requires less than 20 seconds to compute when the simulated time frame is smaller than 100 hours. Comparing to the time that might be necessary to set up a CBHE model with detailed mesh, this is a far more desirable option since the geometry, depth and geothermal gradient can be varied when the proposed analytical model is used to evaluate different $\mathrm{CBHE}$ configurations. Acknowledging the limitations of the proposed methods, we believe the strengths in computational time should also be emphasized, particularly within the scope of this paper.

\section{Conclusion}

We developed a bottom-up analytical model of a coaxial borehole heat exchanger that takes into account of the geothermal gradient when modeling its longer-term performances. Using the analytical model to generate corresponding thermal resistance, we were able to show how the thermal performances of coaxial borehole heat exchangers vary under different configurations and flow rates for the CBHEs. According to our results, a very effective method that improves the performance of CBHEs is to insulate the inner pipe - thus effectively increasing the resulting temperature at the outlet at CBHE. The temperature drop within the internal (central) tube could go down to below 1 degree Celsius if the right combination of design variables is selected, which in turn risks the geothermal depletion of targetted boreholes as heat extraction normalized by depths goes beyond $50 \mathrm{~W} / \mathrm{m}$.

Future research was deemed necessary on both the direction of understanding the relationship between precise temperature profile modeling and the long term performance of CBHEs - and how this might be used to shift the energy demand during peak heating/cooling scenarios. Additional discussions on how to use CBHEs indirect heating/cooling mode could help to quantify the exergetic efficiency, or the COP of overall system in contrast to conventional heat pump mode could also be considered valuable, in particular 
for boreholes that are deep enough to provide temperature output that is suitable for direct heating in the winter.

\section{References}

Acuña, J. (2013). Distributed thermal response tests : New insights on U-pipe and Coaxial heat exchangers in groundwater-filled boreholes. DIVA.

Acuña, J. and B. Palm (2013, September). Distributed thermal response tests on pipe-in-pipe borehole heat exchangers. Applied Energy 109, 312-320.

Beier, R. A., J. Acuña, P. Mogensen, and B. Palm (2013). Borehole resistance and vertical temperature profiles in coaxial borehole heat exchangers. Applied Energy 102, 665 - 675.

DiPippo, R. (2004, October). Second Law assessment of binary plants generating power from lowtemperature geothermal fluids. Geothermics 33(5), $565-586$.

Gnielinski, V. (1976). New equations for heat and mass transfer in the turbulent flow in pipes and channels. International Chemical Engineering 16, 359-368.

GUILlaume, F. (2011). Analysis of a novel Pipe in Pipe Coaxial Borehole Heat Exchanger. Ph. D. thesis, KTH Industrial Engineering and Manage, Stockholm, Sweden.

Ladislaus Rybach and Robert J. Hopkirk (1995, May). Shallow and deep borehole heat exchangers - achievements and prospects. Florence, Italy.

Palm, B., P. Mogensen, J. Acuña, and R. A. Beier (2014). Transient heat transfer in a coaxial borehole heat exchanger. Geothermics 51, 470-482.

Rybach, L., W. J. Eugster, R. J. Hopkirk, and B. Kaelin (1992, October). Borehole heat exchangers: longterm operational characteristics of a decentral geothermal heating system. Geothermics 21(5-6), 861-867. 